\title{
NOUVELLE
}

\section{Gènes stemness et bioingénierie des greffons cutanés}

\section{Focus sur KLF4}

Nicolas 0. Fortunel, Michèle T. Martin

\author{
Laboratoire de génomique et radiobiologie \\ de la kératinopoïèse, CEA/DRF/IBFJ/IRCM, \\ 2 rue Gaston Crémieux, 91000 Évry, France ; Inserm U967, \\ Fontenay-aux-Roses; Université Paris-Diderot, \\ Université Paris-Saclay, France. \\ nicolas.fortunel@cea.fr \\ michele.martin@cea.fr
}

> Le travail de notre laboratoire publié dans la revue Nature Biomedical Engineering [1] identifie le facteur de transcription KLF4 comme un régulateur du statut de cellule souche dans le lignage des kératinocytes humains. Maintenir l'expression de ce facteur à un faible niveau par répression pharmacologique constitue une approche prometteuse pour favoriser l'amplification ex vivo de deux types de cellules d'intérêt pour la thérapie cellulaire cutanée: les cellules souches et progéniteurs kératinocytaires natifs issus d'épiderme adulte, utilisés depuis plus de trois décennies pour la bioingénierie des greffons cliniques, et les kératinocytes générés par différenciation ciblée de cellules souches embryonnaires (CSE), qui font actuellement l'objet d'explorations pour le développement de biopansements cutanés.

\section{La question biomédicale}

L'épiderme humain est naturellement doté de remarquables capacités de renouvellement et de régénération, dues à la présence de cellules souches au sein de sa couche basale. Ces capacités ont permis l'obtention d'organoïdes de peau en culture ainsi que le développement clinique des greffes autologues, notamment pour le traitement des grands brûlés [2, 3]. Plus récemment, une approche combinant thérapies cellulaire et génique a permis la régénération de l'épiderme entier d'un patient atteint d'une génodermatose ${ }^{1}$ [4]. La bioingénierie de grandes surfaces de substituts cutanés nécessite une phase d'expansion ex vivo massive des kératinocytes du patient, durant laquelle la préservation des cellules souches constitue un point critique pour assurer la pérennité des greffes. L'étude des acteurs moléculaires impliqués dans le maintien de cellules souches kératinocytaires fonctionnelles constitue donc une étape nécessaire à la conception des prochaines générations de greffons cutanés. Ces nouvelles générations de greffons pourraient utiliser un matériel cellulaire complémentaire des kératinocytes natifs, puisque les kératinocytes produits par orientation ciblée de CSE ou de cellules souches pluripotentes induites (CSPi) sont capables de générer des organoïdes cutanés [5] (Figure 1). L'efficacité de cette source alternative de kératinocytes dépendra de la robustesse des méthodes de leur production et de la possibilité d'obtenir des kératinocytes à l'état de précurseurs immatures.

\footnotetext{
${ }^{1}$ Groupe hétérogène de maladies génétiques de la peau.
}

\section{Le candidat KLF4}

Le facteur de transcription KLF4 (Kruppel-like factor 4) n'est pas un inconnu dans le domaine des cellules souches. La famille KLF est impliquée dans l'autorenouvellement des CSE, et KLF4 est utilisé dans le procédé original de reprogrammation de cellules somatiques vers un état CSPi [6]. On connaissait également l'implication de KLF4 dans les processus de différenciation terminale des kératinocytes de l'épiderme de souris et son importance pour la mise en place de la fonction barrière de cet épithélium. En revanche, l'exploration des fonctions de KLF4 dans les cellules souches tissulaires natives constitue un pan peu exploré de la biologie de ce facteur. En nous fondant sur des cribles transcriptomiques réalisés sur des sous-populations de précurseurs kératinocytaires humains, enrichies en cellules souches ou en progéniteurs, nous avons posé comme hypothèse que KLF4 était impliqué dans la régulation des cellules souches de l'épiderme, et particulièrement de leur capacité régénératrice.

\section{Étude de la fonction de KLF4 dans les kératinocytes natifs adultes La fonction régulatrice du facteur de transcription KLF4 a tout d'abord été étu-}




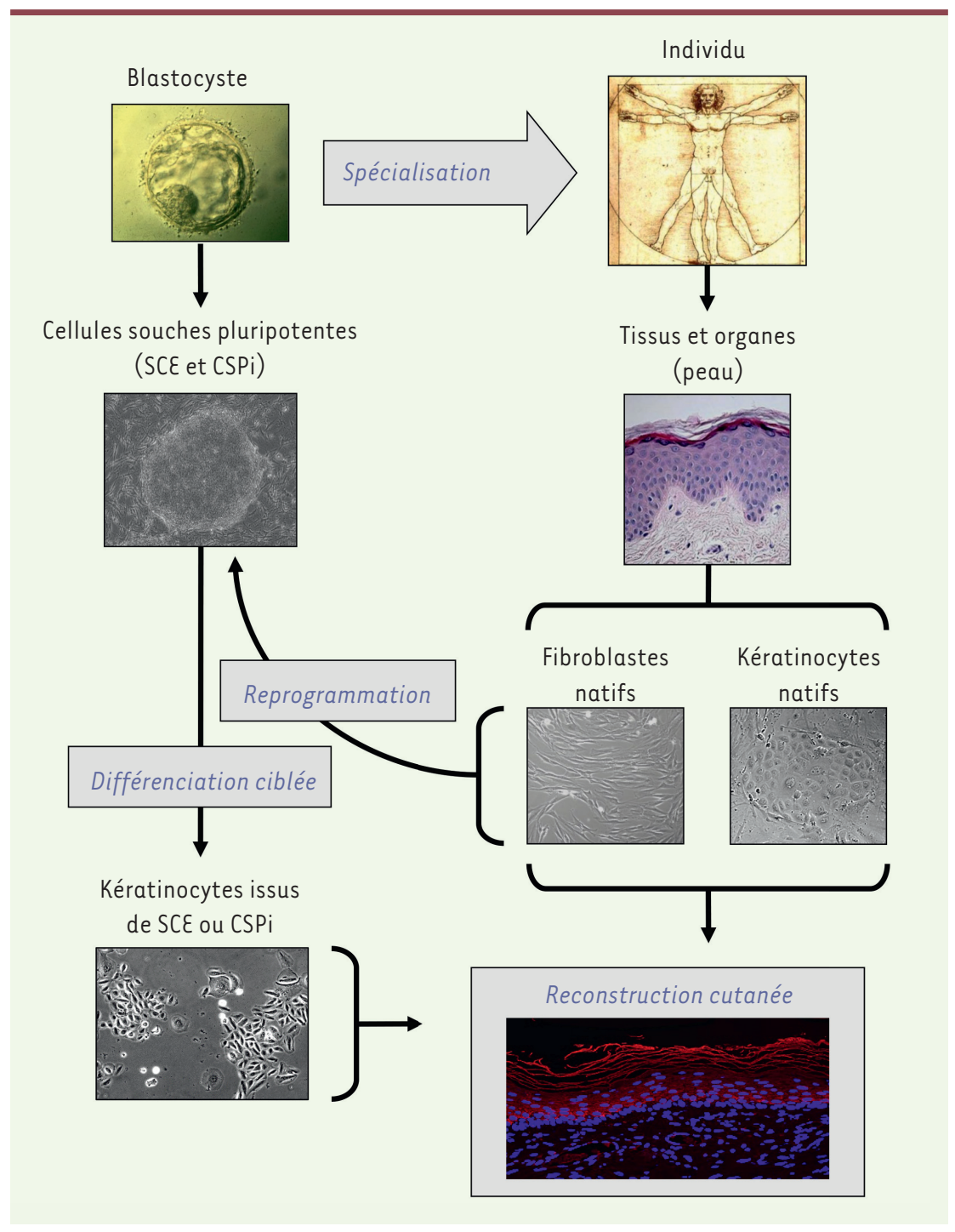

Figure 1. Différentes origines de kératinocytes d'intérêt pour la reconstruction cutanée. Les kératinocytes natifs extraits de biopsies de peau adulte constituent à ce jour la source majeure de kératinocytes pour les utilisations médicales, puisqu'ils sont utilisés depuis plus de trois décennies pour la bioingénierie de greffons destinés au traitement des grands brûlés, et plus récemment pour la thérapie génique. Les kératinocytes produits par différenciation ciblée de cellules souches pluripotentes sont actuellement évalués comme source complémentaire pour le développement de biopansements cutanés. Les cellules souches embryonnaires (CSE) peuvent être différenciées en kératinocytes capables de reconstruire un épiderme. Les cellules souches induites à la pluripotence (CSPi), qui sont obtenues par reprogrammation de cellules adultes (par exemple fibroblastes ou kératinocytes cutanés), peuvent également être différenciées en kératinocytes.

diée dans le modèle des holoclones, qui correspond à la descendance clonale de cellules souches kératinocytaires, maintenue et amplifiée en culture [7]. Dans le cadre d'une approche de génomique fonctionnelle, des holoclones présen- clonalité et de cultures à long terme a permis de montrer qu'une réduction du niveau d'expression de KLF4 préserve le statut de précurseurs kératinocytaires et favorise leur autorenouvellement. Cet effet se traduit par le maintien d'un niveau élevé de nombreux marqueurs caractéristiques d'un état immature de ces cellules, dont l'intégrine- $\alpha 6$, et par une augmentation de l'expansion cellulaire en culture bidimensionnelle. De plus, la capacité régénératrice des cultures de kératinocytes $\left[K L F 4^{K D}\right]$ est supérieure à celle d'une culture de kératinocytes $\left[K L F 4^{W T}\right]$. Ce gain a été démontré d'une part par une meilleure production d'organoïdes en culture, et, d'autre part, par la capacité de substituts cutanés humains à assurer une prise de greffe itérative chez la souris [8]. Lors d'un premier cycle de xénogreffe, l'aptitude des kératinocytes $\left[K L F 4^{\mathrm{WT}}\right]$ et $\left[K L F 4^{\mathrm{KD}}\right]$ s'est avérée équivalente, indiquant une absence d'effet délétère de la répression de KLF4 sur l'organogenèse épidermique. $\varepsilon n$ revanche, lorsque les cellules sont extraites de greffons primaires afin d'assurer un second cycle de reconstruction épidermique et de xénogreffe, le taux de succès observé avec les kératinocytes $\left[K L F 4^{K D}\right]$ est trois fois supérieur à celui des kératinocytes $\left[K L F 4^{W T}\right.$, indiquant un meilleur maintien à long terme de cellules souches kératinocytaires fonctionnelles après répression de KLF4.

\section{Données mécanistiques}

Afin d'expliquer les effets bénéfiques obtenus par inhibition de KLF4, les profils d'expression génique de kératinocytes $\left[\mathrm{KLF}^{\mathrm{WT}}\right]$ et de kératinocytes [KLF4 $\left.{ }^{\mathrm{KD}}\right]$ ont été caractérisés par séquençage du transcriptome et comparés. Cette analyse a permis de mettre en évidence une modulation des voies de signalisation du TGF- $\beta 1$ (transforming growth factor $\beta 1$ ) et de Wnt, qui sont centrales pour la biologie des cellules souches. Nous avons notamment détecté une répression globale de la voie du TGF- $\beta 1$ dans les kératinocytes $\left[K L F 4^{K D}\right]$, et en particulier de son axe ALKI (activin receptor-like 


\section{A}

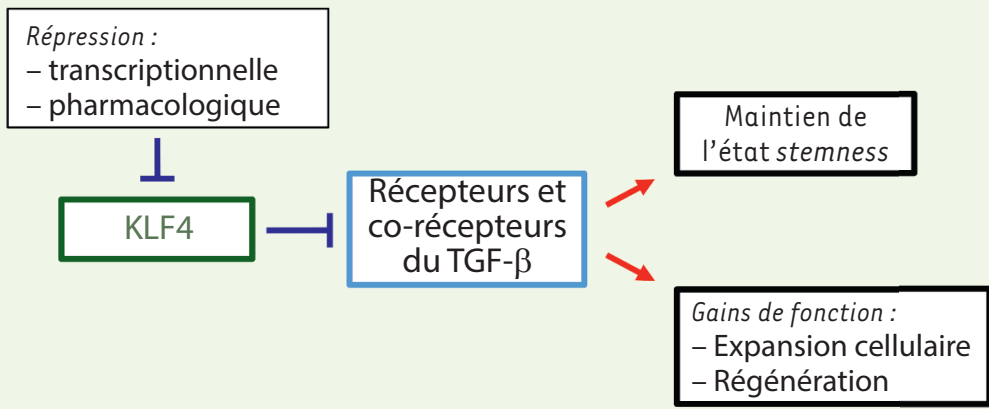

B

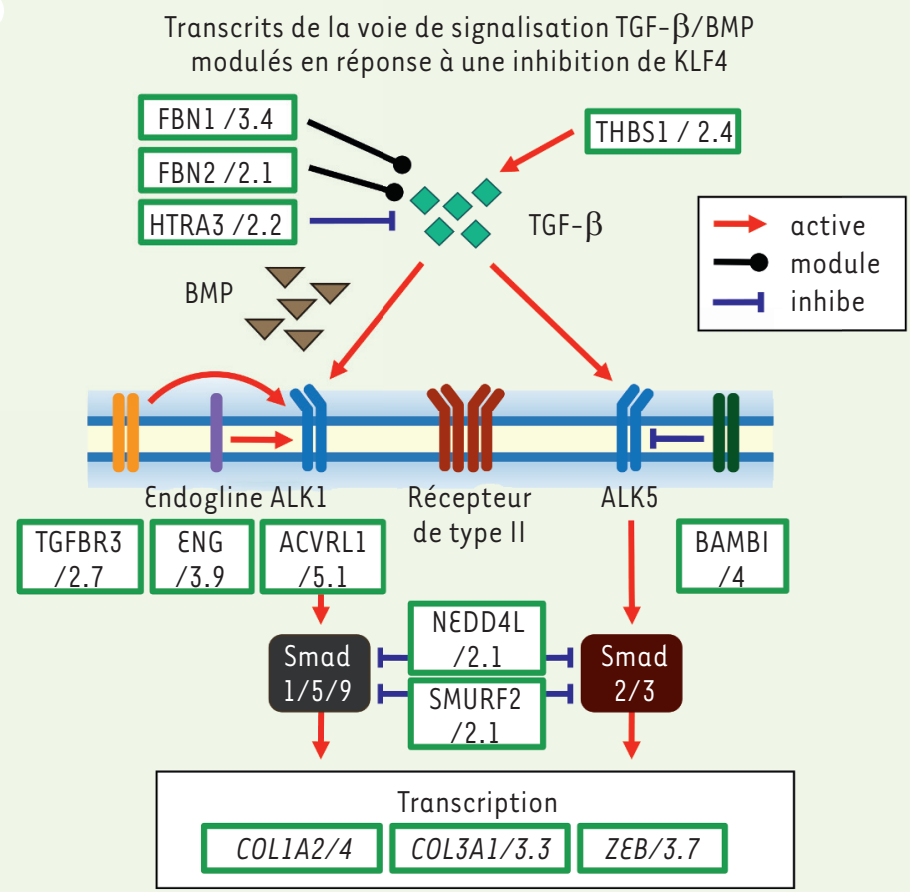

Figure 2. La répression de KLF4 impacte les voies de signalisation du TGF- $\beta$. La comparaison des profils transcriptionnels de kératinocytes $\left[\mathrm{KLF}^{\mathrm{WT}}\right]$ et $\left[\mathrm{KLF}^{\mathrm{KD}}\right]$, caractérisés par séquençage (RNA-seq), montre que la répression de KLF4 diminue l'expression de nombreux gènes codant des effecteurs des voies de signalisation du TGF- $\beta$ (A), incluant des modulateurs extracellulaires, des récepteurs membranaires et des composants intracellulaires $(B)$. Les chiffres indiqués correspondent au différentiel d'expression dans les kératinocytes $\left[K L F 4^{K D}\right]$ versus $\left[K L F 4^{\mathrm{WT}}\right]$.

kinase 1)/Smadl/5/9 (Figure 2). Comme le TGF- $\beta 1$ est connu pour induire la différenciation des précurseurs kératinocytaires, nous avons exploré la relation fonctionnelle entre ce facteur et KLF4. Nous montrons que la répression de KLF4 permet aux kératinocytes de résister à l'effet différenciateur du TGF- $\beta 1$ et de mieux conserver les caractéristiques de cellules souches.
De la recherche fondamentale aux modèles précliniques

Pour nous approcher de conditions applicables en clinique, nous avons exploré les effets d'une répression transitoire de KLF4 sur des kératinocytes issus de l'ensemble de la couche basale de l'épiderme [9], plus représentatifs des cellules utilisées pour la génération des greffons que les holo- clones. Le traitement de kératinocytes par des petits ARN interférents (siARN) ciblant KLF4 a permis de montrer qu'une inhibition transitoire spécifique est efficace pour promouvoir le maintien de caractères associés à un état de cellule souche. L'étape suivante a été l'obtention d'une inhibition transitoire par voie pharmacologique. En effet, un traitement par la kenpaullone ${ }^{2}$ permet de diminuer l'expression de KLF4, ce qui augmente la capacité clonogénique et la croissance des kératinocytes. Sur le plan de la biosécurité, le séquençage de l'exome des kératinocytes n'a pas détecté d'effet délétère de cette répression de KLF4 sur l'intégrité de leur génome.

\section{Extrapolation aux kératinocytes issus} de cellules souches pluripotentes Afin d'élargir la portée de ce travail, nous avons étudié la possibilité d'appliquer la stratégie de la répression de KLF4 aux kératinocytes dérivés de cellules souches embryonnaires (Ker-CSE). Bien que ces kératinocytes possèdent la capacité de reconstruire un épiderme fonctionnel [5], ils ne présentent pas l'ensemble des caractéristiques des cellules souches kératinocytaires, et leur capacité de prolifération en culture est notamment très réduite. La kenpaullone s'est également avérée efficace pour réprimer KLF4 dans ces cellules et pour induire un effet bénéfique sur la prolifération cellulaire, similaire à celui obtenu avec les kératinocytes natifs. $\varepsilon n$ effet, nous avons montré que les Ker-CSE traités avec cette molécule ont un phénotype plus immature, qui se traduit par un gain de croissance en culture bidimensionnelle et un gain de qualité pour la génération d'épidermes tridimensionnels.

\section{Perspectives}

Cette étude a permis d'identifier le facteur de transcription KLF4 comme

${ }^{2}$ Un inhibiteur de la glycogène synthase kinase $3 \beta$ (GSK-3 $\beta$ ) et des kinases dépendant des cyclines (CDK). 


\section{A}

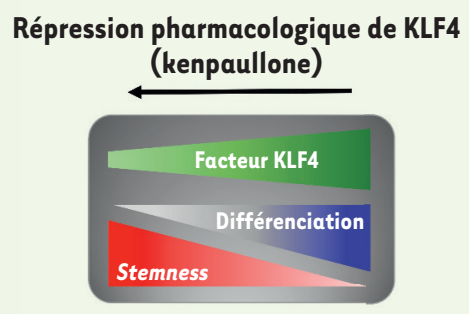

B

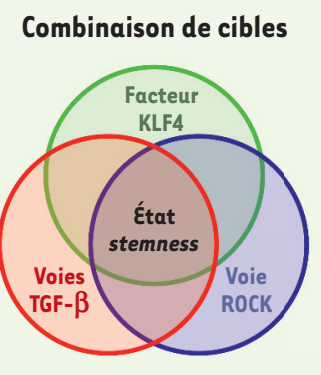

Figure 3. Améliorer l'amplification cellulaire ex vivo par l'utilisation de molécules promotrices d'un état de cellule souche (stemness). Les travaux de notre laboratoire ont permis d'identifier le facteur de transcription KLF4 comme une cible pertinente (A), qui pourrait être utilisée de manière combinée avec d'autres cibles candidates (B), afin de mettre au point des milieux de culture de composition définie pour une utilisation biomédicale.

un régulateur du statut de cellule souche dans le lignage des kératinocytes humains, impliquant notamment un contrôle des voies de signalisation du TGF- $\beta$ l. Maintenir l'expression de ce facteur à un niveau faible par une répression pharmacologique constitue une approche prometteuse pour favoriser l'amplification ex vivo de kératinocytes natifs de l'épiderme humain ou dérivés de cellules souches pluripotentes, deux stratégies d'intérêt pour la thérapie cellulaire cutanée. Par ailleurs, l'expansion des kératinocytes à des fins médicales utilisant actuellement des milieux de culture non définis [10], et notamment du sérum bovin, les instances de règlementation demandent la mise en œuvre de milieux mieux définis et sans composés d'origine animale. La substitution des composés non définis par des molécules actives promotrices du caractère souche, telles que des inhibiteurs de KLF4 ou des voies de signalisation TGF- $\beta$ ou ROCK (Rho-associated, coiled-coil domain kinase), comme cela a été récemment proposé [11], constitue donc un progrès attendu de l'ingénierie cellulaire (Figure 3 ). $\diamond$

When stemness genes meet skin graft bioengineering: focus on KLF4

\section{REMERCIEMENTS}

Nous remercions les co-auteurs de la publication originale. Ces travaux ont bénéficié du soutien logistique et financier du CEA, de l'Inserm (UMR 967), et du Genopole-Évry, ainsi que de financements de la délégation générale de l'armement (DGA), du fonds unique interministériel (FUIAAP13) et du conseil général de l'Essonne (consortium STEMSAFE), de l'AFM-Téléthon, et de fonds de l'union européenne (RISK-IR, FP7, n³23267).

\section{LIENS D'INTÉRÊT}

Les auteurs déclarent n'avoir aucun lien d'intérêt concernant les données publiées dans cet article.

\section{RÉFÉRENCES}

1. Fortunel NO, Chadli L, Coutier J, et al. KLF4 inhibition promotes the expansion of keratinocyte precursors from adult human skin and of embryonic-stem-cellderived keratinocytes. Nat Biomed Eng 2019 ; 3 : 985 97.

2. Gallico GG, O'Connor NE, Compton CC, et al. Permanent coverage of large burn wounds with autologous cultured human epithelium. N Engl J Med 1984 ; 311 : 448-51.

3. Ronfard V, Rives JM, Neveux Y, et al. Long-term regeneration of human epidermis on third degree burns transplanted with autologous cultured epithelium grown on a fibrin matrix. Transplantation $2000 ; 70: 1588-98$.

4. Hirsch $\mathrm{T}$, Rothoeft $\mathrm{T}$, Teig N, et al. Regeneration of the entire human epidermis using transgenic stem cells. Nature 2017 ; 551 : 327-32.

5. Guenou H, Nissan X, Larcher F, et al. Human embryonic stem-cell derivatives for full reconstruction of the pluristratified epidermis: a preclinical study. Lancet $2009 ; 374: 1745-53$.

6. Takahashi K, Tanabe K, Ohnuki M, et al. Induction of pluripotent stem cells from adult human fibroblasts by defined factors. Cell 2007 ; $131: 861-72$.

7. Fortunel NO, Cadio $\varepsilon$, Vaigot P, et al. Exploration of the functional hierarchy of the basal layer of human epidermis at the single-cell level using parallel clonal microcultures of keratinocytes. Exp Dermatol 2010 ; $19:$ 387-92.

8. Fortunel NO, Bouissou-Cadio E, Coutier J, Martin MT. Iterative three-dimensional epidermis bioengineering and xenografting to assess long-term regenerative potential in human keratinocyte precursor cells. Methods Mol Biol 2020 ; 2109 : 155-67.

9. Fortunel NO, Chadli L, Bourreau $\varepsilon$, et al. Cellular adhesion on collagen: a simple method to select human basal keratinocytes which preserves their high growth capacity. Eur J Dermatol 2011 ; Suppl 2 : 12-20.

10. Alexaline MM, Trouillas M, Nivet M, et al. Bioengineering a human plasma-based epidermal substitute with efficient grafting capacity and high content in clonogenic cells. Stem Cells Transl Med $2015 ; 4: 643-54$.

11. Zhang C, Lee HJ, Shrivastava A, et al. Long-term in vitro expansion of epithelial stem cells enabled by pharmacological inhibition of PAKl-ROCK-Myosin II and TGF- $\beta$ signaling. Cell Rep $2018 ; 25: 598-610$.

\section{LA FONDATION PREMUP : UN OPÉRATEUR DE TERRAIN EN PÉRINATALITÉ RECONNU POUR SON EXCELLENCE ET SON INTERDISCIPLINARITÉ}

La Fondation de coopération scientifique PremUp, unique en Europe, intervient sur la prévention du handicap à la naissance, par la protection de la santé de la femme enceinte et du nouveau-né.

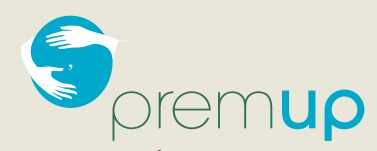

FONDATION DE COOPÉRATION SCIENTIFIQUE SUR LA GROSSESSE ET LA PRÉMATURITÉ

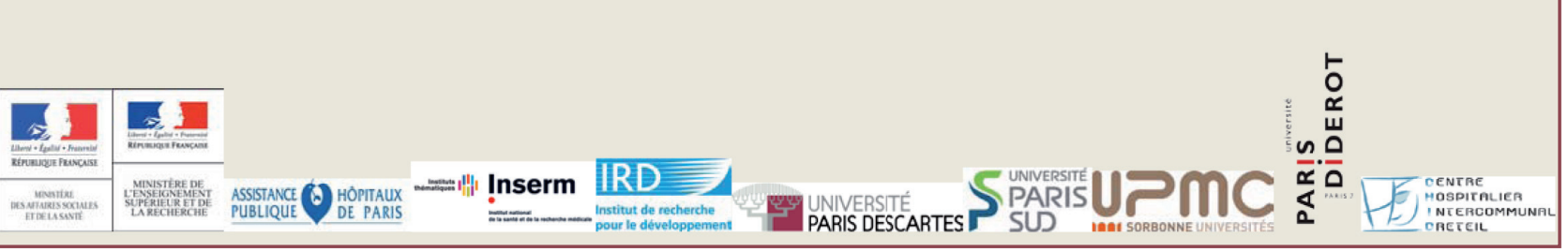

